\title{
Pediatric Malignant Peripheral Nerve Sheath Tumour of the Radial Nerve
}

\author{
Mohammed F. Shamji, Bibiana Purgina, Trevor Flood, Jean Michaud, Enrique C.G. Ventureyra
}

Can. J. Neurol. Sci. 2010; 37: 524-527

Malignant peripheral nerve sheath tumours (MPNST) are a group of malignant tumours that arise from cells that are differentiating or have differentiated toward those of the peripheral nerve sheath. ${ }^{1}$ The heterogeneity of these cellular components and stages of differentiation have historically lead to difficulty in defining the diagnosis and behaviour of these lesions, with terms such as malignant schwannoma, neurofibrosarcoma, and neurogenic sarcoma variably used to define the histopathology. Indeed, most MPNSTs do exhibit some degree of Schwannian differentiation, however this histological feature may be absent and alternately fibroblastic or perineurial cellular phenotype may predominate, with further metaplastic tissue characterizing $15 \%$ of lesions. ${ }^{2}$ These lesions usually arise de novo with rare reports of malignant degeneration from known or suspected benign disease, with known association of these lesions with Type I neurofibromatosis (NF1). ${ }^{3,4}$

The necessity of correct histological differentiation from benign tumours is based on the need for complete resection with negative tumour margins portending favourable survival prognosis, and the additional reported benefit of adjuvant therapies such as intraoperative electron radiotherapy, brachytherapy, and postoperative irradiation. ${ }^{5,6}$ Further, Anghileri and coworkers ${ }^{7}$ reviewed the literature about such lesions finding high likelihood of local recurrence (27 to $49 \%$ at five years) and substantial but variable cause specific mortality (23 to $81 \%$ at five years), reflecting heterogeneous clinical behaviour that will require further investigation to best delineate patients expected to have favourable therapeutic result.

We report the case of a seven-year-old patient with biopsy demonstrated MPNST of the radial nerve who underwent complete local excision and no adjuvant therapy. This patient exhibits long-term survival, followed without secondary intervention for the last 22 years, with no evidence of local or metastatic disease recurrence. The histopathological features of pediatric MPNST will be reviewed from both the literature and this patient to assess features that may have suggested this prognosis from the index evaluation.

\section{Case Report}

We present the case of a seven-year-old girl with a past medical history significant for Prader-Willi syndrome, vesiculoureteral reflux, and chronic pyelonephritis. She is righthanded, and presented in March 1986 with a ten-month history of a non-tender left upper extremity mass lesion, with a diameter of $5 \mathrm{~cm}$ and located deep within the triceps brachii muscle tissue. The mass was both soft and mobile on examination, with no associated pain, nor any motor or sensory deficit in the extremity. Imaging studies are unavailable, but the reports include:
1. X-Ray revealed a soft tissue mass lateral to the mid-shaft of the humerus, differentiated from subcutaneous fat and without bony involvement.

2. Computed tomography revealed a soft tissue mass lateral to the humeral shaft, containing patchy areas of fat, and without contrast enhancement.

3. Bone scan revealed minimal area of increased radiotracer activity adjacent to the left humerus.

Open surgical exploration of this lesion by the orthopaedic surgery service revealed it to arise from the radial nerve, with the decision taken to biopsy the mass and defer definitive treatment until the pathology was established. Based on the malignant appearance from the biopsy sample and the uncertainty at that time (1986) of the clinical behaviour of this tumour, expert opinion was sought for further histological evaluation and thereafter also for the optimal treatment plan. The pathology was again delineated as a malignant schwannoma, with surgical consultation advocating for aggressive and radical resection in the form of upper extremity amputation. Consequently, two treatment paradigms were offered to the patient. First, the possibility of forequarter amputation was offered as most likely to achieve definitive cure maximizing likelihood of negative margins and minimizing possibility of local recurrence and metastatic disease. Second, the possibility of local excision alone was offered with benefit of retained upper extremity function, but with higher risk of local recurrence and metastatic spread, mandating close clinical follow-up.

The patient's parents opted for local resection, and the tumour that measured $5.0 \mathrm{~cm}$ in its major diameter was totally excised by the attending neurosurgeon (ECGV, senior author) with a clear plane of cleavage from the proximal radial nerve. Intraoperatively, the nerve fascicle from which the tumour originated was identified and transected, and there was no gross evidence of residual tumour capsule or tumour invasion into the radial nerve following excision. Further, the tumour itself seemed encapsulated that facilitated complete surgical resection, and there was no evidence of invasion into surrounding soft tissue. The radial nerve was observed to be redundant from substantial stretching, but nonetheless in anatomic continuity. Following

\footnotetext{
From the Division of Neurosurgery (MFS, ECGV), Division of Pathology (BP, TF, JM), Children's Hospital of Eastern Ontario and University of Ottawa; Division of Neurosurgery (MFS), The Ottawa Hospital, Ottawa, Ontario, Canada.

Received November 23, 2009. Final Revisions Submitted February 4, 2010. Correspondence to: Enrique Ventureyra, Division of Neurosurgery, Children's Hospital of Eastern Ontario, 401 Smyth Road, Ottawa, Ontario, K1H 8L1, Canada.
} 


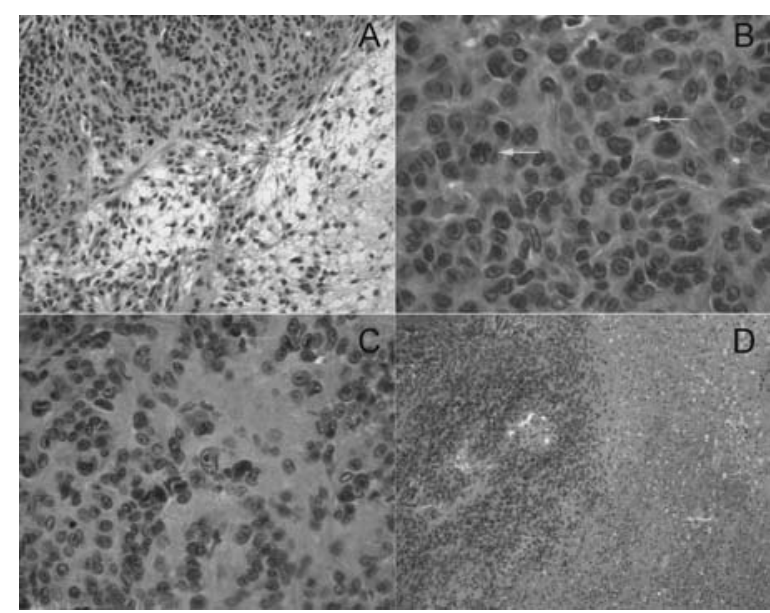

Figure 1: Histological evaluation of radial nerve tumour. Panel A (200x) shows regions of the tumour that present a benign appearance containing cellular Antoni A areas and looser, myxoid Antoni B areas. Panel B (600x) shows abnormal mitotic figures (arrows) containing pleomorphic nuclei with irregular and overlapping nuclear contours. Panel C (400x) shows atypical epithelioid cells that are variable in size and shape and that contain prominent nucleoli with a coarse chromatin pattern. Panel D (100x) shows the abrupt transition from a necrotic area to viable tumour cells, with the necrotic zone containing abundant debris, ghost cells, and inflammatory cells.

consultation with the oncology service, contemporaneous adjuvant therapy was not offered particularly given the complete lesion resection.

The excised surgical specimen consisted of ovoid tan coloured soft tissue mass measuring $5.0 \times 3.2 \times 2.3 \mathrm{~cm}$ with a mass of 25.0 grams. The lesion was grossly well-encapsulated. Cut sections demonstrated focal areas of haemorrhage and necrosis. Microscopic examination is shown in Figure 1, and revealed similar histologic features in both the biopsy and the excised specimen. A capsule of variable thickness surrounds and separates a neoplasm from an adjacent large calibre nerve, with no tumour penetration through the capsule. There are multiple areas where the cells display atypical features including increased nuclear: cytoplasmic ratio, pleomorphism, and nuclear hyperchromasia. Numerous mitotic figures are also observed, some of which are abnormal in microtubular disorganization and excessive DNA content. There are large zones of necrosis $(\sim$ $30 \%$ of lesion), with haemorrhage and cystic degeneration. Other fields show areas of dense cellularity alternating with hypocellular myxoid regions, a finding more consistent with Antoni A and B patterns observed in benign schwannomas. The neoplastic cells arise in a fibrillar background and are uni- or bipolar with indistinct cell borders, and arrange radially around vessels frequently forming pseudo-rosettes.

For more definitive analysis, the stored lesion specimen was evaluated by contemporary immunohistochemical techniques (Figure 2) including S-100, vimentin, Ki67, glial fibrillary acidic protein (GFAP), cytokeratin AE1.3, epithelial membrane antigen (EMA) and smooth muscle actin (SMA), protein gene product 9.5 (PGP9.5), and p53 glycoprotein. Primary antibodies were streptavidin-linked, with detection using biotin-linked horseradish peroxidise. The key findings are shown in Figure 2 and include diffuse positivity for S-100 supported the diagnosis of schwannoma, with patchy GFAP and cytokeratin positivity also suggesting glial origin. The Ki67 level of overall 5\% (but in various foci approaching 50\%) in combination with abnormal mitoses further supports malignant lesion behaviour, as did the strong nuclear staining for p53 and focal strong staining for PGP9.5.

The postoperative course was complicated by an immediate but transient radial nerve palsy, most profoundly affecting wrist extensors. This deficit lasted four months during which physiotherapy was administered, after which slow and sustained clinical improvement was observed. After 11 months follow-up, no objective motor or sensory deficit could be discerned. At a recent encounter 22 years after tumour excision, symmetric upper extremity sensory and motor function are appreciated with no clinical evidence of recurrent disease.

\section{Discussion}

The biological aggressiveness of MPNST is well described with poorer clinical outcomes governed by the need for wide resection and negative surgical margins, high rates of local disease recurrence, and the side effects of adjuvant therapy. A review of current cases in the literature is summarized in Table 1 alongside descriptions of current prognostic factors. Current management is focussed on as complete surgical resection as possible, although application of neo-adjuvant therapy in the form of pre-operative chemotherapy and radiotherapy may

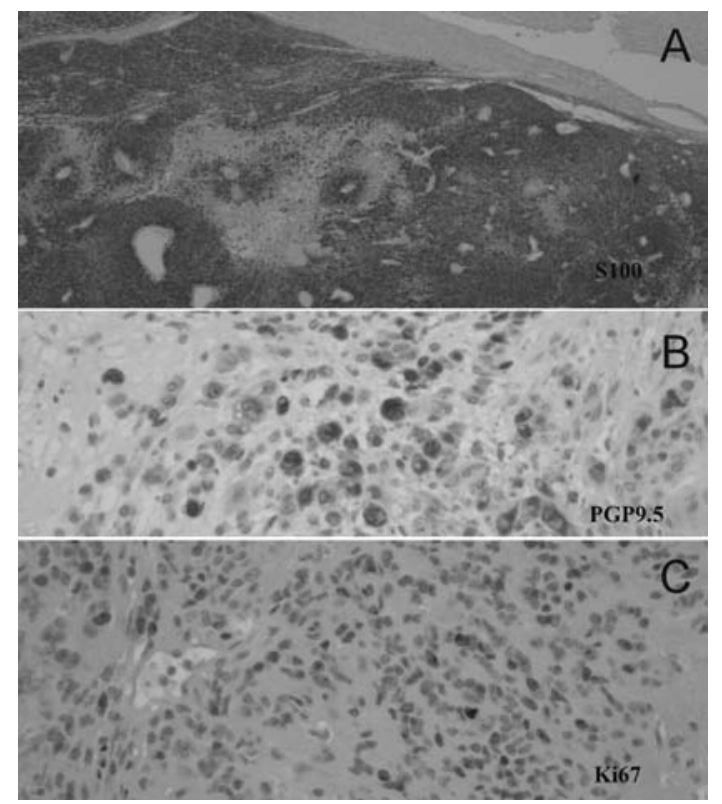

Figure 2: Immunohistochemical evaluation of radial nerve tumour. Panel A (S100, 20x) shows strong and diffuse immunoreactivity with an intact perineural capsule and vascular spaces. The non-immunoreactive regions represent zones of necrosis. Panel B (PGP9.5, 200x) reveals strong staining in more than 50\% of tumour cells. Panel C (Ki67, 200x) reveals significant staining of nearly $50 \%$ of tumour cells in one of the most active foci of the lesion. Average staining for this antigen in this tumour was approximately $5 \%$. 
provide for lesion shrinkage and facilitate operative intervention.

Carli and coworkers ${ }^{8}$ report on the largest series of 167 cases of pediatric MPNST, with diagnosis at a median age of 11 years. The lesions were split between axial $(60 \%)$ and peripheral (40\%), with $17 \%$ of patients having associated diagnosis of NF1. Overall survival of $51 \%$ was observed at five years, with regression analysis describing adverse prognostic factors of tumour size above $5 \mathrm{~cm}$, tumour invasiveness, comorbid NF1 diagnosis, and axial location. Meis and coworkers ${ }^{9}$ also describe a similar large series of 78 cases of pediatric MPNST, with diagnosis made at a median of ten years-of-age. The lesions were similarly split between axial (54\%) and peripheral (46\%) locations, with $21 \%$ of patients having associated diagnosis of NF1. Median survival was estimated at 45 months with $50 \%$ of patients exhibiting local recurrence at 12 months and $50 \%$ of patients exhibiting metastases at 24 months. In regression analysis, adverse prognostic factors include larger tumour size, age over seven years at presentation, and comorbid NF1. A series of 20 pediatric patients reported by Casanova and coworkers ${ }^{10}$ describes a median age of 12 years, of whom $29 \%$ had associated NF1 diagnosis, and one third of patients having extremity tumours. Survival at ten years was $41 \%$, with adverse prognostic factors including subtotal tumour resection and tumour size above $5 \mathrm{~cm}$.

Substantial histological and immunohistochemical variability has been reported for MPNST, with immunophenotypic analysis the patient reported herein summarized in Table 2. In the series of 78 patients reported by Meis and coworkers ${ }^{9}, 56 \%$ and $62 \%$ of patients exhibit S-100 and vimentin positivity respectively. The S-100 antigen is a calcium-binding protein that is not exclusive of MPNST, with positivity seen in schwannomas, ependymomas, astroglial tumours, and melanoma, but that does suggest neural differentiation of the tumour components particularly when diffusely positive, with melanomatous lesions exhibiting a more patchy distribution. Vimentin is a major structural component of intermediate filaments, with positivity in nervous system tumours suggesting immaturity or early differentiation of the cellular components. Further, cytokeratin AE1.3 positivity is observed in a quarter of patients, with actin positivity in $40 \%$ and Leu7 positivity in $45 \%$. Actin positivity in these lesions may represent the early stage of cellular differentiation or invasion into the surrounding muscle tissue, but is also described in patients whose tumours have rhabdomyoplastic components. ${ }^{11-13}$ Significant Leu7 positivity is an expected finding with myelin-associated glycoprotein as the antibody target, although cell surface reactivity with natural killer cells is also well known. Cytokeratin AE1.3 is commonly used to screen for epithelial components of tumour, particularly in poorly differentiated malignant lesions, although reactivity against both glial and non-glial tumours that express GFAP is well known and consequently may have limited value in discerning the malignant potential of this subgroup of lesions. Of note, in this series of patients, the only histological feature portending adverse prognosis in the multivariate regression analysis was tumour necrosis in excess of $25 \%$, with no immunohistochemical features providing additional predictive value. Liapis and coworkers ${ }^{14}$ describe a series of 10 MPNSTs with particular attention to labelling of p53 and Ki67 antigens. Immuno-positivity for p53 was observed in $60 \%$ of cases, with such result generally indicating the presence of a mutant inactive protein product incapable of negatively regulating the cell cycle as a tumour suppressor gene. This is because the mutant product exhibits greater longevity than the wild-type protein that undergoes rapid dephosphorylation turnover. ${ }^{15,16}$ The Ki67 antigen provided greater sensitivity with immunopositivity in all (10/10) cases of MPNST, and only one of their 12 cases of benign neurofibromas utilized for comparison. Based on this result, the authors advocated for use of this target as assistive in differentiating between benign and malignant peripheral nerve sheath tumours, particularly in lesions with atypical or pleomorphic cellular phenotype.

The tumour documented in this report demonstrated a strong Schwannian component with diffuse staining for S-100, though patchy GFAP and cytokeratin AE1.3 positivity were also observed. Immunoreactivity for both GFAP and cytokeratin AE1.3 are expected given the suspected glial cell origin, but neither helps estimate the tumour aggressiveness. Malignant features of nuclear pleomorphism and atypia, tumour necrosis, and 5\% Ki67 labelling with focal regions of much higher expression were instrumental in helping establish the diagnosis of MPNST, but unremarkable for the purpose of understanding the benign clinical course that she has experienced. The very

Table 1: Clinical series of pediatric malignant nerve sheath tumours

\begin{tabular}{|c|c|c|c|c|c|c|}
\hline Author & $\mathbf{N}$ & $\begin{array}{l}\text { Median } \\
\text { Age (yrs) }\end{array}$ & Gender & NF-1 & Survival & $\begin{array}{l}\text { Adverse Prognostic } \\
\text { Factors }\end{array}$ \\
\hline Meis (9) & 78 & 10 & & $17 \%$ & median: 48 months & $\begin{array}{l}\text { Size }>5 \mathrm{~cm} \\
\text { NF1 } \\
\text { Axial location } \\
\text { Tumour invasiveness }\end{array}$ \\
\hline Carli (8) & 167 & 11 & $\begin{array}{l}\text { M } 83 \\
\text { F } 84\end{array}$ & $21 \%$ & 5-year: $51 \%$ & $\begin{array}{l}\text { Large size } \\
\text { Age }>7 \text { yr } \\
\text { NF1 } \\
\text { Tumour necrosis }>25 \%\end{array}$ \\
\hline $\begin{array}{l}\text { Casanova } \\
(10)\end{array}$ & 24 & 12 & $\begin{array}{l}\text { M } 13 \\
\text { F } 11\end{array}$ & $29 \%$ & 10-year: $41 \%$ & $\begin{array}{l}\text { Subtotal resection } \\
\text { Size }>5 \mathrm{~cm}\end{array}$ \\
\hline Liapis (14) & 10 & 11 & $\begin{array}{l}\text { M } 4 \\
\text { F } 6\end{array}$ & $60 \%$ & NR & NR \\
\hline $\begin{array}{l}\text { Current } \\
\text { (Shamji) }\end{array}$ & 1 & 7 & $\mathrm{~F}$ & No & Alive at 22 year follow-up & N/A \\
\hline
\end{tabular}




\section{Table 2: Immunohistochemical characteristics of the reported case of radial nerve malignant peripheral nerve sheath tumour}

\begin{tabular}{|c|c|c|}
\hline Antigen & Result & Significance \\
\hline S-100 & Strong, diffuse & $\begin{array}{l}\text { Calcium-binding protein consistent with } \\
\text { Schwannian cell of origin }\end{array}$ \\
\hline Vimentin & Strong, diffuse & $\begin{array}{l}\text { Intermediate filament reflecting early stage of } \\
\text { differentiation }\end{array}$ \\
\hline GFAP & Focal, cytoplasmic & $\begin{array}{l}\text { Intermediate filament reflecting glial cell of } \\
\text { origin }\end{array}$ \\
\hline AE1.3 & Strong, diffuse, cytoplasmic & $\begin{array}{l}\text { Potential confounded by GFAP } \\
\text { immunopositivity }\end{array}$ \\
\hline SMA & Focal near capsule & No capsular invasion \\
\hline EMA & Focal invading into capsule & No capsular invasion \\
\hline Leu7 (CD57) & Intermediate, diffuse, cytoplasmic & Neuron specific adhesion molecule \\
\hline Ki67 & $5 \%$ positivity & $\begin{array}{l}\text { Mitotically active tumour, supporting } \\
\text { malignant phenotype with }>5 \%\end{array}$ \\
\hline p53 & Strong, nuclear & Loss of cell cycle regulation \\
\hline PGP9.5 & Strong, focal & $\begin{array}{l}\text { Neuron specific protein reflecting cellular } \\
\text { metabolic activity }\end{array}$ \\
\hline
\end{tabular}

good biological behaviour of this reported tumour is best explained by several factors (young age and location in an extremity with early detection, no association with neurofibromatosis, small tumour size, absence of capsular invasion permitting resection margins free of tumour, and significant S100 positivity and relatively low Ki-67 indicating a lower malignant grade even if significant necrosis and abnormal mitoses were present.

Despite advances in both diagnosis and management, patients with MPNST are exposed to challenging prognosis with high rates of local recurrence and poor long-term survival. Children with such lesions may have worse prognosis, with the largest series documenting median survivals in this population of less than two years. We report the highly unusual case of a patient whose radial nerve tumour was identified at age seven as malignant schwannoma based on the degree of nuclear atypia, cellular pleomorphism, and patchy areas of necrosis. Following complete local tumour excision only with no radical surgery (limb amputation) or adjuvant treatment, she remains alive and well-functioning at long-term followup of 22 years. The histology and malignant nature of the tumour was confirmed using current immunohistochemical techniques as MPNST, with expected S-100, GFAP, and cytokeratin AE1.3 positivity, and 5\% Ki67 labelling index. Oddly, no single specific features predict or explain the favourable biological behaviour. Nevertheless, this points to the significant heterogeneity in the biological aggressiveness of MPNSTs thus motivating further investigation into features prognosticating favourable postoperative course. Reporting histological characteristics and immunophenotype of cases with either early demise and long-term survival may help identify such features.

\section{REFERENCES}

1. Hirose T, Scheithauer BW, Sano T. Perineurial malignant peripheral nerve sheath tumour (MPNST): a clinicopathologic, immunohistochemical, and ultrastructural study of seven cases. Am J Surg Pathol. 1998;22(11):1368-78.

2. Ducatman BS, Scheithauer BW, Piepgras DG, Reiman HM, Ilstrup DM. Malignant peripheral nerve sheath tumours. A clinicopathologic study of 120 cases. Cancer. 1986;57(10):2006-21.
3. Evans DG, Baser ME, McGaughran J, Sharif S, Howard E, Moran A. Malignant peripheral nerve sheath tumours in neurofibromatosis 1. J Med Genet. 2002;39(5):311-4.

4. D'Agostino AN, Soule EH, Miller RH. Sarcomas of the peripheral nerves and somatic soft tissues associated with multiple neurofibromatosis (Von Recklinghausen's Disease). Cancer. 1963;16:1015-27.

5. Wong WW, Hirose T, Scheithauer BW, Schild SE, Gunderson LL. Malignant peripheral nerve sheath tumour: analysis of treatment outcome. Int J Radiat Oncol Biol Phys. 1998;42(2):351-60.

6. Ferner RE, Gutmann DH. International consensus statement on malignant peripheral nerve sheath tumours in neurofibromatosis. Cancer Res. 2002;62(5):1573-7.

7. Anghileri M, Miceli R, Fiore M, Mariani L, Ferrari A, Mussi C, et al. Malignant peripheral nerve sheath tumours: prognostic factors and survival in a series of patients treated at a single institution. Cancer. 2006;107(5):1065-74.

8. Carli M, Ferrari A, Mattke A, Zanetti I, Casanova M, Bisogno G, et al. Pediatric malignant peripheral nerve sheath tumour: the Italian and German soft tissue sarcoma cooperative group. J Clin Oncol. 2005;23(33):8422-30.

9. Meis JM, Enzinger FM, Martz KL, Neal JA. Malignant peripheral nerve sheath tumours (malignant schwannomas) in children. Am J Surg Pathol. 1992;16(7):694-707.

10. Casanova M, Ferrari A, Spreafico F, Luksch R, Terenziani M, Cefalo $\mathrm{G}$, et al. Malignant peripheral nerve sheath tumours in children: a single-institution twenty-year experience. J Pediatr Hematol Oncol. 1999;21(6):509-13.

11. Karpuz V, Letovanec N, Von Hochstetter A, Joris F. [Malignant peripheral nerve sheath tumour with rhabdomyoblastic differentiation and glandular component]. Ann Pathol. 2000;20 (1):62-5.

12. Rose DS, Wilkins MJ, Birch R, Evans DJ. Malignant peripheral nerve sheath tumour with rhabdomyoblastic and glandular differentiation: immunohistochemical features. Histopathology. 1992;21(3):287-90.

13. Stasik CJ, Tawfik O. Malignant peripheral nerve sheath tumour with rhabdomyosarcomatous differentiation (malignant triton tumour). Arch Pathol Lab Med. 2006;130(12):1878-81.

14. Liapis H, Marley EF, Lin Y, Dehner LP. p53 and Ki-67 proliferating cell nuclear antigen in benign and malignant peripheral nerve sheath tumours in children. Pediatr Dev Pathol. 1999;2(4):37784.

15. Baas IO, Mulder JW, Offerhaus GJ, Vogelstein B, Hamilton SR. An evaluation of six antibodies for immunohistochemistry of mutant p53 gene product in archival colorectal neoplasms. J Pathol. 1994;172(1):5-12.

16. Humphrey PA. p53: mutations and immunohistochemical detections, with a focus on alterations in urologic malignancies. Arch Pathol Lab Med. 1994;47:579-606. 